\title{
Inmersión en agua salina templada. Adaptación cardiovascular en jóvenes sanos
}

\author{
Valeria SCANDELLA ${ }^{(1)}$, María BURUCHAGA ${ }^{(1)}$, Marta GIACOMINO$^{(1)}$, \\ Daniel de MICHELE ${ }^{(1)}$
}

${ }^{(1)}$ Departamento de Posgrado, Facultad de Ciencias de la Salud, Universidad Nacional de Entre Ríos, Pablo Lorenz s/n, 3260 - C. del Uruguay, Entre Ríos (Argentina).

demichele@fcs.uner.edu.ar

Recibido: 30-06-11

Aceptado: 15-07-11

\section{Resumen}

Objetivo: Determinar la respuesta cardiovascular adaptativa, durante y después de la inmersión del cuerpo en agua salina templada en jóvenes sanos.

Material y Métodos: Población: 20 jóvenes sanos (20-25 años) seleccionados al azar. Inmersión: agua templada $\left(38^{\circ} \mathrm{C}\right)$, nivel del esternón. Determinación de la presión arterial y frecuencia cardíaca en: a) Pre-inmersión; b) Inmersión: de pie a los 10, 20, 30 minutos; c) Post-inmersión: después de dejar agua y en reposo a 10, 20 y 30 minutos. El agua fue clasificada previamente como: a) hipertermales $\left(38^{\circ} \mathrm{C}\right)$; b) fuerte mineralización; c) Los iones predominantes son: cloruro, sulfato, bicarbonato de sodio; d) extremadamente duras. Resultados: Presión arterial: La presión arterial media pre inmersión fue 114/70. La inmersión de 30 minutos, provocó una disminución promedio del $30 \%$ de los valores de presión diastólica y el $45 \%$ de los valores de presión sistólica en comparación con el valor inicial. Los valores mínimos se obtuvieron a los 30 minutos de inmersión (media: 80/40). 30 minutos después de salir del agua, la presión arterial media para toda la población fue 99.7/63 $\mathrm{mm} \mathrm{Hg}$. Hubo un patrón de recuperación de los valores tensionales basales después de salir del agua, pero incluso a los 30 minutos en pos inmersión, los valores de referencia no se han alcanzado. Frecuencia cardíaca: Hubo una elevación de la frecuencia cardíaca leve pero sostenida hasta 30 minutos de inmersión (media de 97,3 latidos / min) con restauración de los valores basales a los 30 minutos después de la inmersión. No se detectaron efectos adversos.

Conclusiones: La inmersión en agua templada por 30 minutos $\left(38^{\circ} \mathrm{C}\right.$, y con iones predominantes como el sodio, cloruros, sulfatos y bicarbonato, en el establecimiento de Villa Elisa, Argentina), produjo una notable disminución de la presión arterial y aumento del ritmo cardíaco en 20 jóvenes sanos. No se detectaron efectos adversos.

Palabras claves: Inmersión, Termalismo, Balneoterapia 


\title{
Saline warm water immersion. Cardiovascular adaptation in healthy young
}

\begin{abstract}
Objective: Determine cardiovascular adaptative response in healthy young people, during and after saline warm water immersion.

Material and Methods: Population: 20 randomly selected healthy youngs (20-25 years old). Immersion: warm water $\left(38^{\circ} \mathrm{C}\right)$, at sternal level. Determination of blood pressure and heart rate at: a) Pre-immersion; b) Immersion: standing at 10, 20, 30 minutes; c) Pos-immersion: after leaving water in rest at 10, 20 and 30 minutes. Water was previously categorized as: a) hyperthermal $\left(38^{\circ} \mathrm{C}\right)$; b) strong mineralization; c) prominent ions: chloride, sulphate, bicarbonate, sodium; d) extremely hard water.

Results: Blood Pressure: Pre-immersion mean blood pressure was 114/70. Immersion of 30 minutes, provocated an average decrease of $30 \%$ of diastolic values and $45 \%$ of systolic values compared with baseline. The minimum values were obtained at 30 minutes of immersion (mean: 80/40). 30 minutes after leaving the water, blood pressure average for the entire population was $99.7 / 63 \mathrm{~mm} \mathrm{Hg}$. There were tensional values recovery after leaving the water, but even at 30 minutes post-immersion, baseline values were not reached. Heart rate: There was a slight but sustained heart rate elevation until 30 minutes of immersion (mean 97.3 beats/min) with restoration of baseline values at 30 minutes after immersion. No adverse effects were detected.

Conclusions: Warm water immersion $\left(38^{\circ} \mathrm{C}\right.$ and predominant ions; sodium, chloride, sulfate and bicarbonate, in Villa Elisa Spa, Argentina) of 20 healthy young by 30 minutes produced remarkable decreased blood pressure and increased heart rate. No adverse effects were detected.
\end{abstract}

Key words: Immersion, Health resort medicine, Balneotherapy

\section{REFERENCIA NORMALIZADA}

Scandella V, Burruchaga M, Giacomino M, de Michele D. Inmersión en agua salina templada. Adaptación cardiovascular en jóvenes sanos. (Saline warm water immersion. Cardiovascular adaptation in healthy young). Anal Hidrol Med, 2011, vol. 4, 33-43

\section{INTRODUCCION}

La inmersión en aguas templadas con diversas concentraciones de sales, es una práctica que se ha popularizado con el desarrollo del Termalismo y el Spa. Desde los '90 y gracias al lugar ganado por el concepto calidad de vida, la práctica del termalismo se ha incrementado notablemente, contando entre sus usuarios a personas afectadas por síndromes articulares o cutáneos crónicos, especialmente artrosis, 
fibromialgia y psoriasis. Sin embargo, la enorme mayoría de usuarios son personas sanas, que acuden a los complejos termales o spas con fines recreacionales, dando lugar a una corriente denominada Termoludismo. Esta modalidad se ha desarrollado notablemente en Europa y también en nuestro país.

La fisiología de la inmersión, indica que las modificaciones cardiovasculares inducidas por el redireccionamiento del volumen sanguíneo hacia el tórax, se siguen inmediatamente de ajustes en la frecuencia cardíaca y la presión arterial; lo habitual es la aparición de taquicardia y descenso de la presión arterial, más o menos proporcional a la temperatura del agua y el tiempo de inmersión. A la inversa, la inmersión en agua fría, produce vasoconstricción, aumento de la resistencia periférica, tendencia a la bradicardia y aumento de los valores de la presión arterial. Este fenómeno se verifica incluso, sumergiendo únicamente una mano en agua fría y ha sido reportado hace 60 años ${ }^{1}$.

Como la práctica del Termalismo cuenta con por lo menos 25 siglos, hay un cuerpo de conocimientos empíricos acumulados y relatos anecdóticos sobre modificaciones de la presión arterial ante la inmersión en aguas salinas templadas. En base a tales conocimientos empíricos, se han formulado indicaciones, contraindicaciones y probables riesgos de tal práctica. No obstante ello, hay pocos reportes sistemáticos sobre la adaptación cardiovascular fisiológica ante la inmersión breve en fluidos termales.

Existe en cambio mucha información sobre fisiología de la inmersión, proveniente de la investigación aeroespacial y el buceo durante los 60 y 70, cuando se intentaba emular las situaciones de ingravidez mediante la inmersión del cuerpo en agua.

Es dificultoso extrapolar el conocimiento obtenido a partir de esas investigaciones, ya que se obtuvo sobre la base de estudios que analizaban la fisiología humana en situación de largas exposiciones a la inmersión y otras situaciones extremas, que no coinciden con la breve inmersión que importa la práctica actual del termalismo con fines lúdicos y/o terapéuticos.

Considerando que millones de personas al año, practican el Termalismo, parece oportuno investigar específicamente la respuesta tensional a estímulos físicos (efecto hidrostático e inmersión en agua a temperaturas superiores a la corporal) y químicos (exposición a un medio externo hipertónico).

Sabemos que una rápida vasodilatación de los vasos de la superficie corporal, es la respuesta adaptativa frente a cualquier fuente de calor que modifique la temperatura a la que el cuerpo está sometido. Sin embargo, aun no comprendemos completamente todos los fenómenos que ocurren en la inmersión en agua templada salina en personas sanas. 


\section{MATERIAL Y METODOS}

\section{Muestra:}

De un universo de usuarios del establecimiento termal de Villa Elisa, Entre Ríos a lo largo de un día $(n=719)$, se tomo una muestra aleatoria simple de 20 individuos para el estudio (de 10 hombres y 10 mujeres), considerando los siguientes criterios de inclusión: individuos sanos, entre 20 y 25 años, sin antecedentes patológicos, normotensos, no fumadores, libres de fármacos en últimas 3 semanas y adhesión al consentimiento informado diseñado para la prueba.

\section{Caracterización fisicoquímica del agua empleada para las pruebas de inme- sión:}

Las propiedades fisicoquímicas del agua mineral utilizada, fueron previamente estudiadas por nuestro grupo de investigación y publicadas oportunamente ${ }^{2}$.

Según los standard de Hidrología Medica ${ }^{3}$ las aguas del establecimiento en que se realizó el estudio fueron clasificadas como sigue:

- Por su temperatura; Hipertermales $\left(38^{\circ} \mathrm{C}\right)$.

- Por contenido de minerales: de Mineralización Fuerte (17 gr/l)

- Por los iones que contienen: Cloruradas (6,8 gr/l), Sódicas $(5,5 \mathrm{gr} / 1)$ y Sulfatadas $(3,5 \mathrm{gr} / 1)$.

- Por su dureza: Extremadamente Duras

- Por su radiactividad: No radiactivas

\section{Protocolo:}

Se diseñó contemplando en todos sus términos la Declaración de Helsinki modificada $(2000)^{4}$. Las pruebas se realizaron en el mismo día, por lo cual las condiciones climáticas y locales del agua salina fueron las mismas para todos los casos estudiados.

El estudio incluyó mediciones de;

1. El estudio incluyó mediciones de;

a) pre inmersión en reposo,

b) inmersión de pie e inmóvil con agua hasta el nivel del manubrio esternal a 10,20 y 30 minutos y

c) pos inmersión en reposo a los 10, 20 y 30 minutos

2. frecuencia cardiaca en las mismas condiciones que la presión arterial 


\section{RESULTADOS}

Hubo modificaciones de la Presión Arterial y la frecuencia cardíaca tras la inmersión en agua templada salina en el $100 \%$ de los pacientes examinados. Los valores medios de presión arterial y frecuencia cardiaca (hombres y mujeres) para cada momento de la prueba se exhiben en la Tablas y Figuras I y II.

Tabla I - Valores medios de Tensión Arterial durante la prueba

\begin{tabular}{|c|c|c|c|}
\hline \multicolumn{2}{|c|}{ Presión } & $\begin{array}{c}\text { Diastólica } \\
\text { mm } \mathbf{H g}\end{array}$ & $\begin{array}{c}\text { Sistólica } \\
\text { mm } \mathbf{H g}\end{array}$ \\
\hline \multicolumn{2}{|c|}{ Pre inmersión } & 114 & 70 \\
\hline \multirow{3}{*}{ 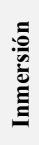 } & $10 \mathrm{~min}$ & 94,5 & 53,7 \\
\hline & $20 \mathrm{~min}$ & 88 & 44,2 \\
\hline & $30 \mathrm{~min}$ & 80,7 & 40 \\
\hline \multirow{3}{*}{ 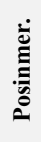 } & $10 \mathrm{~min}$ & 103 & 62 \\
\hline & $20 \mathrm{~min}$ & 97,5 & 60,3 \\
\hline & $30 \mathrm{~min}$ & 99,7 & 63 \\
\hline
\end{tabular}

Figura 1 - Valores medios de Tensión Arterial durante la prueba

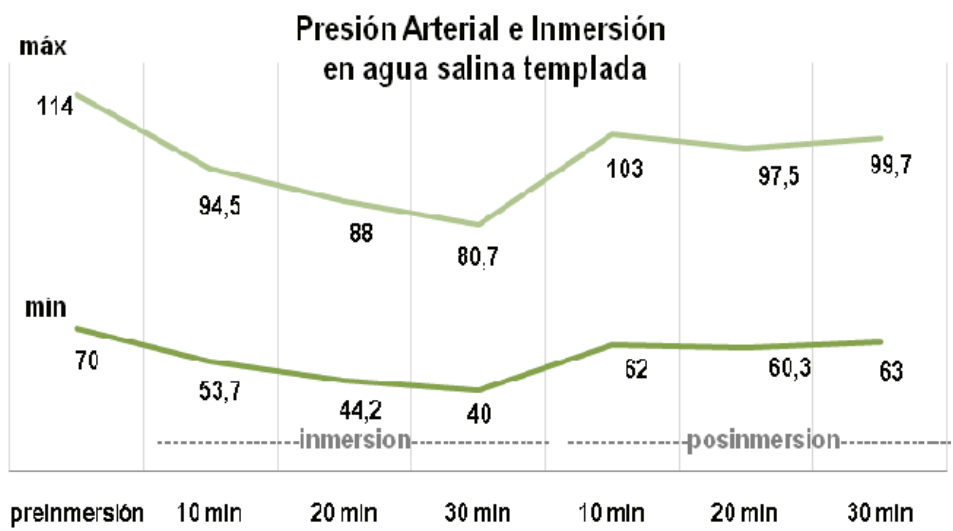

Ocurrieron pequeñas diferencias en el comportamiento de los valores tensionales entre hombres y mujeres durante la inmersión, pero estos no fueron estadísticamente significativos y parecen más vinculados a los niveles basales de tensión arterial que a efectos de la inmersión misma. 
El patrón de reducción de los valores de presión diastólica y sistólica durante la inmersión así como la recuperación ulterior incompleta en la post-inmersión aparece en una curva similar en ambos sexos.

Tabla II - Valores medios de Tensión Arterial durante la prueba

\begin{tabular}{|c|c|c|}
\hline \multicolumn{2}{|c|}{ Frecuencia Cardíaca } & latidos/min \\
\hline \multicolumn{2}{|c|}{ Pre inmersión } & 71,5 \\
\hline \multirow{3}{*}{ 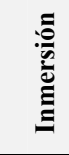 } & $10 \mathrm{~min}$ & 83,3 \\
\hline & $20 \mathrm{~min}$ & 91 \\
\hline & $30 \mathrm{~min}$ & 97,3 \\
\hline \multirow{3}{*}{ 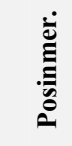 } & $10 \mathrm{~min}$ & 76 \\
\hline & $20 \mathrm{~min}$ & 71,5 \\
\hline & $30 \mathrm{~min}$ & 69 \\
\hline
\end{tabular}

Figura 2 - Valores medios de Frecuencia Cardíaca durante la prueba

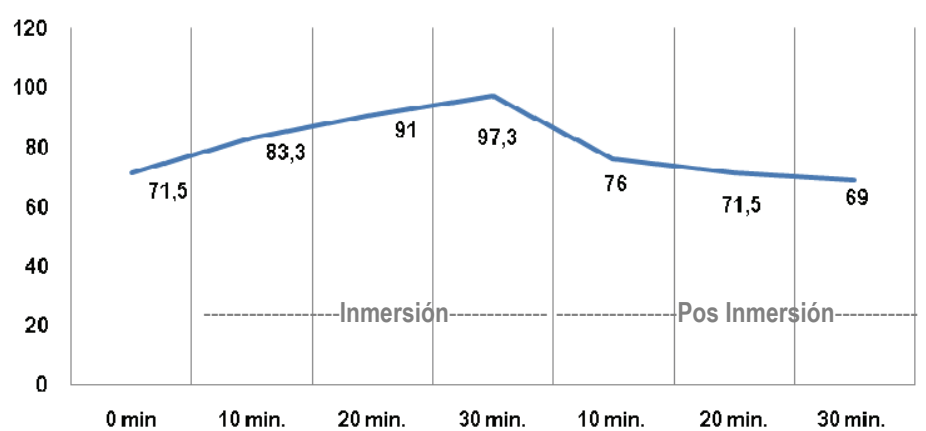

La presión diastólica media de toda la población estudiada, osciló entre un máximo de 70 y un mínimo de $40 \mathrm{~mm} \mathrm{Hg}$.

El valor absoluto más bajo luego de 30 minutos de inmersión fue de $30 \mathrm{~mm} \mathrm{Hg}$. La presión sistólica media de toda la población estudiada, osciló entre un máximo de 114 y un mínimo de $80,7 \mathrm{~mm} \mathrm{Hg}$. El valor absoluto más bajo fue de $70 \mathrm{~mm} \mathrm{Hg}$. Todos los individuos del estudio, volvieron a elevar su presión arterial en el período de pos inmersión, pero a los 30 minutos luego de finalizada la inmersión, ninguno alcanzó los valores basales detectados al inicio de la prueba. 
No aparecen efectos adversos, molestias, signos de hipotensión ni de otro tipo en ningún paciente tras la inmersión de 30 minutos en agua templada salina, ni siquiera en los tres pacientes (dos mujeres y un hombre) que presentaron valores de presión arterial tan bajos como 70/30 $\mathrm{mm} \mathrm{Hg}$.

La curva de cambio de la frecuencia cardíaca muestra una pendiente ascendente, suave y continua hasta los 30 minutos de inmersión cuando se ubicó entre 74 y 118 latidos/minuto para los 20 participantes del estudio (media de 97,3 mm Hg).

A partir de allí, los valores comienzan a descender retornando a los niveles de base a los 30 minutos posteriores a la inmersión.

\section{DISCUSION}

Han sido reportados repetidamente en la bibliografía internacional, los efectos cardiovasculares de la inmersión del cuerpo humano en agua templada. Uno de los primeros informes fue comunicado hace casi 90 años ${ }^{5}$.

Más de veinte años atrás, Weston demostró utilizando ecodoppler que en una población de "16 personas normales" el flujo cardiaco aumenta un $30 \%$ tras la inmersión en agua a $33^{\circ} \mathrm{C}$, alcanzando a un $121 \%$ a $39^{\circ} \mathrm{C}^{6}$. En dicho estudio también se informa que la resistencia periférica disminuye notablemente a medida que aumenta la temperatura del agua. Cambios en la frecuencia cardiaca, con tendencia a la taquicardia también fueron comunicados.

Otros autores, han cuantificado la modificación de la resistencia periférica durante la inmersión de agua templada e informan que aquella cae un $37 \%$ en agua a $34.5^{\circ} \mathrm{C}$ y $32 \%$ en agua a $30^{\circ} \mathrm{C}^{7}$.

Los hallazgos de nuestro estudio, coinciden con los de Weston en cuanto a patrones de presión arterial y la frecuencia cardiaca frente a la inmersión en agua mineral templada.

La notable vasodilatación periférica que genera la inmersión en agua a temperatura cercana a la corporal, parece tener un rol central en el descenso de la presión arterial.

Si bien en nuestro estudio no hemos calculado resistencia periférica, es obvio que ha ocurrido un descenso marcado, revelado por la caída de los valores tensionales en presencia de taquicardia relativa.

Está demostrado previamente en la literatura internacional que el volumen minuto cardíaco aumenta en individuos normales y en portadores de insuficiencia cardiaca congestiva crónica tras la inmersión en agua templada ${ }^{8-9-10-11-12-13-14}$.

Por otro lado, si el resultado final de la inmersión en agua templada es una caída de la presión arterial, la importante elevación de uno de los componentes mayores en la ecuación de presión arterial - el volumen/min- sugiere una fuerte disminución 
del otro componente: la resistencia periférica. Esta, se reduce progresiva y sostenidamente a medida que la temperatura del agua se eleva.

Estudios provenientes del campo de la fisiología del buceo y de aguas a diferentes temperaturas y de las investigaciones realizadas desde la medicina aeroespacial (NASA), tratando de generar ambientes similares a la ingravidez durante los años '60 y '70, han sido fuente de conocimiento en este campo para la Hidrología Médica actual ${ }^{15-16-17-18-19-20-21-22}$.

Sabemos que la inmersión del cuerpo en agua a 34 o $35^{\circ} \mathrm{C}$, provoca un desplazamiento inmediato de sangre de los miembros y un aumento en el volumen de sangre intratorácica ${ }^{15}$.

Rápidamente aumenta el gasto cardíaco a través de un mayor volumen diastólico final y el volumen de eyección debido al efecto del estiramiento y mejoría de la fuerza contráctil del músculo cardíaco. El estiramiento de la aurícula también da lugar a una diuresis compensatoria ${ }^{23-24}$, lo que en nuestro estudio no pudo detectarse por lo breve de la inmersión.

Gauer \& Henry ${ }^{25}$ sugieren que el aumento del flujo renal ocasionado por el mayor flujo cardíaco ante la inmersión, reduciría el volumen plasmático volviendo el flujo cardíaco a la normalidad.

Ha sido reportado en la literatura que las modificaciones cardiovasculares y renales inducidas por la inmersión en agua templada, ocurren en ambos sexos por igual $^{26-27}$.

Estos reportes coinciden con nuestros hallazgos en la población de 20 jóvenes normotensos (10 hombres y 10 mujeres) estudiados en grupos homogéneos, ya que -como se mostró antes- las modificaciones de la tensión arterial se observaron tanto en hombres como en mujeres sin diferencias estadísticamente significativas.

Pendergast ${ }^{28-29}$ informa que el aumento del gasto cardíaco observado en la inmersión de personas jóvenes está mitigado en los ancianos.

Es claro que el aumento del volumen minuto inducido por la inmersión en agua templada, es contabalanceado en exceso por la caída de la resistencia periférica, única explicación hallada a la respuesta de hipotensión presente en todos los jóvenes del estudio y reportada en la literatura internacional.

Es difícil aceptar que algunos de los fenómenos que ocurren en la inmersión en agua a $34-35^{\circ} \mathrm{C}$ durante 3-6 horas, jueguen un rol en la inmersión de media hora, como la planeada en nuestro estudio. No sabemos si la desviación del volumen sanguíneo de las extremidades hacia el tórax ${ }^{6}$ descripta en la inmersión por horas en aguas templadas, ocurre con exposiciones menores.

Nuestro estudio ha demostrado que la inmersión de jóvenes sanos hasta la altura del manubrio esternal en agua salina templada, provoca modificaciones en la presión arterial.

No existe un patrón universal que permita predecir la magnitud de estos cambios tras la inmersión en agua templada, ya que se conoce que la física y la química del 
agua son variables en cada región, lo que hace prácticamente imposible tener dos aguas minerales iguales.

Evidentemente, la temperatura del agua, el tiempo de inmersión, la presión arterial de base y la salinidad del agua, juegan un rol que será proporcional a la fuerza con que cada componente se expresa en cada tipo de agua. Los hallazgos aquí reportados en cuanto al comportamiento tensional en jóvenes sanos, aparecen como solo aplicables a poblaciones similares que se sometan a inmersión en agua clorurada sódica y sulfatada de Villa Elisa, por 30 minutos.

Se necesita repetir estas pruebas en cada establecimiento termal con diversas poblaciones antes de formular warnings o protocolos generales de inmersión.

\section{DISCUSION}

Los jóvenes sanos, presentan rápida adaptación cardiovascular ante una inmersión en agua salina templada $\left(38^{\circ} \mathrm{C}\right)$ por 30 minutos, con descenso de la presión arterial entre 30 y $45 \%$ de los valores basales.

La frecuencia cardiaca aumenta moderadamente hasta el fin del periodo de inmersión.

Presión arterial y frecuencia cardiaca tienden a volver a valores basales a $30 \mathrm{mi}$ nutos de finalizada la inmersión.

A pesar de los cambios adaptativos cardiovasculares señalados, no parece haber repercusión clínica bajo la forma de efectos colaterales en el grupo estudiado.

Algunos de los los interrogantes que surgen de este estudio, son:

a) ¿qué cambios se producirán por inmersión breve en aguas de diferente temperatura y composición química a la estudiada?,

b) ¿qué ocurrirá en inmersiones de adultos mayores de 25 años, portadores de hipertensión, otras condiciones clínicas y aun sanos?,

c) ¿cuál será la respuesta ante una inmersión prolongada más allá de los 30 minutos de jóvenes sanos y adultos con/sin condiciones asociadas?

Se necesita investigación adicional para diseñar estudios más amplios que intenten responder estas preguntas.

\section{BIBLIOGRAFÍA}

1. Godden J, Roth G. The Changes in the Intra-Arterial Pressure during Immersion of the Hand in Ice-Cold Water. Circulation. 1955;12:963-73.

2. De Michele D, Giacomino M, Belderrain A, Cettour H. Vademecum de Aguas Termales de Entre Ríos. C. del Uruguay; Ediciones Ciencias de la Salud. 2007. 
3. Maraver F, Armijo F. Vademecum II de Aguas Mineromedicinales Españolas. Madrid; Ed. Complutense. 2010.

4. A fifth amendment for the Declaration of Helsinki. The Lancet 2000;356:1123.

5. Bazett H. Studies on the effects of baths on man. I. Relationship between the effects produced and the temperature of the bath. Am J Physiol. 1924;70:41229.

6. Weston CF, O'Hare JP, Evans JM, Corrall RJ. Haemodynamic changes in man during immersion in water at different temperatures. Clin Sci (Lond). 1987;73:613-6.

7. Park K, Choi J, Park Y. Cardiovascular Regulation during Water Immersion. Appl Human Sci. 1999; 18:233-41.

8. Cider A. Immersion in warm water induces improvement in cardiac function in patients with chronic heart failure. Eur J Heart Fail. 2006; 8:308-13.

9. Grüner B. Benefit of warm water immersion on biventricular function in patients with chronic heart failure Cardiovascular Ultrasound. 2009; 7:33.

10. van Tol B, Huijsmans R, Kroon D, Schothorst M, Kwakkel G. Effects of exercise training on cardiac performance, exercise capacity and quality of life in patients with heart failure: a meta-analysis. Eur J Heart Fail. 2006: 8:84150 .

11. Meyer K, Bucking J. Exercise in heart failure: should aqua therapy and swimming be allowed? Med Sci Sports Exerc. 2004: 36(12):2017-23.

12. Schmid $\mathbf{J}$ et al. Influence of water immersion, water gymnastics and swimming on cardiac output in patients with heart failure. Heart. 2007; 93(6):72227.

13. Gabrielsen A et al. Cardiovascular and neuroendocrine responses to water immersion in compensated heart failure. Am J Physiol Heart Circ Physiol. 2000; 279(4):1931-40.

14. Suzuka H, Hirakawa H, Nakashima Y, Hayashida Y. Effect of water immersion on renal sympathetic nerve activity and arterial baroreflex in conscious rabbits with heart failure. Auton Neurosci. 2003; 104(1):39-46.

15. Arborelius M, Balldin U, Lilja C, Lundgren C. Hemodynamic changes in man during immersion with the head above water. Aerospace Medicine. 972; 43:592-98.

16. Bowers JA, Hood, WB, Murray H, Urschel C. Hemodynamic effects of water immersion. Aerospace Medical Association Preprints. 1965; 32-33.

17. Boyer J, Frazer E, Doyle A. The haemodynamic effects of cold immersion. Clin Sci. 1960;19:539-50.

18. Campbell L, Gooden B and Horowitz J. Cardiovascular responses to partial and total immersion in man. J Physiol. 1969:202:239-50. 
19. Carlson LD. Cardiovascular studies during and following simulation and weightlessness. Life Sciences and Space Research V, 7th International Space Science Symposium, Vienna, May 10-18. 1966.

20. Craig AB, Dvorak M. Thermal regulation during water immersion. J Appl Physiol. 1966:21:1577-85.

21. Dawson W, Kottke F, Kubicek W, Olson M, Harstad K, Bearman J, Canner P, Canterbury J. Evaluation of cardiac output, cardiac work and metabolic rate during hydrotherapy and exercise in normal subjects. Arch Phys Med Rehabil. $1965 ; 46: 605-14$.

22. Ferguson J, Chambers C, Schmidt C, Wray W. Psychological aspects of water immersion studies. NADC-MA-6328, U. S. Naval Air Development Center, Johnsville, Pa. 1963.

23. Gabrielsen A, Johansen LB, Norsk P. Central cardiovascular pressures during graded water immersion in humans. Life Sciences Research in Space, Proceedings of the Fifth European Symposium held in Arcachon, France. 1993.

24. Epstein M. Renal effects of head-out water immersion in humans: 15-year update. Physiol Rev.1992;72: 563-621.

25. Gauer OH, Henry JP. Neurohumoral control of plasma volume. In: Cardiovascular Physiology II, edited by Guyton AC, Cowley AW. Baltimore, MD: University Park Press, 1976.

26. Glickman-Weiss E, Cheatham C, Caine M, Blegen M, Marcinkiewicz J, Mittleman K. The influence of gender and menstrual phase on thermosensitivity during cold water immersion. Aviat Space Environ Med. 2000;71:715-22.

27. Watenpaugh D, Pump B, Bie P, Norsk P. Does gender influence human cardiovascular and renal responses to water immersion? J Appl Physiol. 2000; 89: 621-28.

28. Pendergast D, Fisher N, Calkins E. Cardiovascular, neuromuscular, and metabolic alterations with age leading to frailty. J Gerontol. Biol Sci. 1993; 48:617.

29. Pendergast D, Lundgren E. The underwater environment: cardiopulmonary, thermal, and energetic demands. J. Appl. Physiol. 2009;106: 276-83. 